\title{
Muséologies
}

Les cahiers d'études supérieures

\section{Le Temps des Québécois : d'une intention communicationnelle à sa réception}

\section{Audrey Boursaud}

Volume 1, numéro 2, avril 2007

URI : https://id.erudit.org/iderudit/1033609ar

DOI : https://doi.org/10.7202/1033609ar

Aller au sommaire du numéro

Éditeur(s)

Association Québécoise de Promotion des Recherches Étudiantes en Muséologie (AQPREM)

ISSN

1718-5181 (imprimé)

1929-7815 (numérique)

Découvrir la revue

Citer cet article

Boursaud, A. (2007). Le Temps des Québécois : d’une intention communicationnelle à sa réception. Muséologies, 1(2), 70-93.

https://doi.org/10.7202/1033609ar
Résumé de l'article

Audrey Boursaud analyse l'exposition Le Temps des Québécois du Musée de la civilisation, dans le but de démontrer l'adéquation des intentions communicationnelles des concepteurs avec la perception et la recomposition que les visiteurs font de l'exposition grâce aux différents dispositifs scénographiques. d'utilisation que vous pouvez consulter en ligne. 


$$
t_{1}
$$


Audrey Boursaud analyse l'exposition Le Temps des Québécois du Musée de la civilisation, dans le but de démontrer l'adéquation des intentions communicationnelles des concepteurs avec la perception et la recomposition que les visiteurs font de l'exposition grâce aux différents dispositifs scénographiques.

AUDREY BOURSAUD EST TITULAIRE D'UNE MAITRISE ET D'UN DIPLOOME D'ÉTUDES APPROFONDIES EN HISTOIRE AINSI QUE D'UN DIPLÔME D'ÉTUDE SPÉCIALISÉE EN PATRIMOINE. ELLE A ÉTÉ CHARGÉE D'ÉTUDES CHEZ PLANETH CONSULTANTS, FIRME TOURISTIOUE ET CULTURELLE FRANÇAISE. EN 2003, ELLE CHOISIT D'IMMIGRER AU QUÉBEC ET APPREND LAPPROCHE MUSÉALE QUÉBÉCOISE EN OBTENANT SA MAITRISE EN MUSÉOLOGIE (EN 2006). DEPUIS, ELLE RÉALISE DES RECHERCHES ET DES EXPOSITIONS POUR DIVERSES INSTITUTIONS. 
[1]

Cette définition est issue du cours MSL 6104 : “Exposition, interprétation et diffusion". donné par MM. Bernard Schiele et Raymond Montpetit.

[2]

Ce schéma est proposé par l'auteure d'après les définitions issues du livre de DAVALLON, Jean. L'exposition à l'ceuvre, stratégie de communication et médiation symbolique, Paris : l'Harmattan, 1999, 378 p. et du cours MSL 6104 : "Exposition, interprétation et diffusion", donné par MM. Bernard Schiele et Raymond Montpetit.
Une exposition est une structuration spatiale, tridimensionnelle, d'une intention de communiquer une idée, des messages, de la part d'un concepteur/ émetteur à un récepteur, le public. À cette fin, le concepteur construit un espace organisé, le parcours, durant lequel l'exposition composera et délivrera son message communicationnel. Cette structuration s'effectue par le biais de dispositifs qui combinent des artéfacts et d'autres supports médiatiques et interprétatifs, susceptibles d'en porter le contenu et d'en orienter la réception ${ }^{[1]}$. Le concepteur imagine un visiteur type afin de choisir la présentation et l'orientation qui sont censées répondre aux attentes, aux comportements connus et simulés du visiteur ciblé. Une fois l'exposition ouverte, le visiteur modèle, abstrait, n'existe plus. Il devient un visiteur réel, une personne physique avec ses connaissances propres, ses sensibilités, ses modes d'apprentissages (cognitif, auditif, visuels...), ses attentes. Ces visiteurs réels reconstituent le rébus spatial, utilisent ou non les dispositifs, peuvent les détourner de leur usage initial et occulter ou déformer les messages.

FIGURE 1

Schéma théorique de fonctionnement de l'exposition ${ }^{[2]}$

\section{ÉMETTEUR}

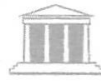

MUSÉE

- CHARGÉ DE PROJET

- ÉQUIPEDE CONCEPTION

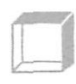

EXPOSITION

CONCEPTION/

UTILISATION

PARCOURS,

DISPOSITIFS, OBJETS...
RÉCEPTEUR

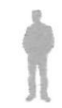

VISITEURS

CONNAISSANCES,

SENSIBILITÉS,

MOTIVATIONS... 
Disciplinaire et chronologique, Le Temps des Québécois est une nouvelle exposition permanente du Musée de la civilisation, inaugurée en juin 2004. Première du genre au musée, elle remplace Mémoires, l'exposition permanente thématique qui était en place depuis dix-sept ans, qui a fait l'objet de nombreuses études et recherches. L'évaluation sommative du Temps des Québécois permettait :

- d'observer son fonctionnement ${ }^{[3]}$;

- de déterminer l'adéquation entre les intentions communicationnelles, comportementales de départ et la réalité de perception, d'utilisation des visiteurs ${ }^{[4]}$;

- de situer Le Temps des Québécois dans son contexte, son insertion au musée;

- de dépeindre l'un des premiers maillons de sa « chaîne de réception ${ }^{[5]}$ ".

L'effet del'inauguration passé, les questions se sont révélées nombreuses : Qui vient visiter cette exposition ? Comment les visiteurs appréhendent-ils le parcours ? Utilisent-ils les différents supports - notamment les films - mis à leur disposition? Comment les visiteurs s'approprient-ils et perçoivent-ils l'exposition Le Temps des Québécois ? Recomposent-ils les intentions communicationnelles déployées dans l'exposition ? Les visiteurs sont-ils satisfaits de leur visite, de leur expérience? Quelle place l'exposition va-t-elle prendre dans le triptyque d'exposition permanente?

\section{[3]}

CHAUMIER, Serge.

"Les méthodes de l'évaluation muséale : quelques repères au sujet des formes et des techniques $\gg$. In. La lettre de I'OCIM, $n^{\circ}$ 65, 1999, p. 13-21; et GOTTESDIENER, Hana et Jean CHAGUIBOFF, Évaluer l'exposition : définitions, méthodes et bibliographie sélective commentée d'études d'évaluation. Paris : Documentation française, 1987, p. 91.

[4]

Cet exercice reste relativement rare au musée; depuis son ouverture, deux expositions permanentes ont fait l'objet d'évaluations approfondies : Mémoires (1988-2005) et Grandir(1999-2000), qui fut retirée peu après l'évaluation, d'ailleurs.

[5]

La chaîne de réception est un concept développé par le théoricien Hans Robert Jauss qui s'intéresse à la succession des réceptions dont une œuvre littéraire fait l'objet au cours du temps. (JAUSS, Hans Robert. Pour une esthétique de la réception. Paris : Gallimard, 1978, 305 p.) Appliquer ce concept pour les expositions a été proposé et réalisé par Cécile Sauvage pour l'exposition Mémoires. (SAUVAGE, Cécile. Mémoires au Musée de la civilisation de Québec. Regards croisés sur une exposition permanente. Musée de la civilisation. Québec : Les cahiers du Musée de la civilisation, 2004, p. 15. 


\section{Présentation de l'exposition et de ses objectifs}

Selon la conception théorique de l'exposition et du rôle du visiteur ci-dessus, l'analyse et la présentation de l'exposition sont des préalables ${ }^{[6]}$ pour apprécier l'adéquation entre les intentions communicationnelles, leurs articulations scénographique et spatiale et la recomposition des visiteurs réels.

D'après la documentation interne ${ }^{[7]}$ et l'analyse de la salle d'exposition, l'objectif principal de l'exposition est de présenter une synthèse de l'histoire des Québécois pour aujourd'hui, en offrant un récit plus positif de l'histoire du Québec que celui qui habite actuellement la mémoire populaire. Dans un second temps, l'équipe de conception souhaite proposer un récit qui

- présente les Québécois comme des acteurs de leur histoire;

- inclut tous les Québécois, quels que soient leur origine ethnique, leur langue, leur religion, le moment de leur arrivée au Québec;

De même, elle peut donner une idée plus précise au lecteur qui n'a pas visité cette exposition.

- dépasse l'histoire politique et intègre l'histoire sociale et culturelle;

- personnifie les acteurs de cette histoire, connus ou anonymes, réels ou tirés de la fiction;

- replace l'histoire du Québec dans la trame de l'histoire internationale;

- valorise des événements passés dont les enjeux sont encore prégnants aujourd'hui.

Ces objectifs servent de fils conducteurs et organisent la stratégie communicationnelle de l'exposition. Plusieurs intentions communicationnelles bénéficient de leurs propres dispositifs : «les voix du passé» sont des bornes d'écoute qui présentent des témoignages de personnages plus ou moins connus et réels. Elles permettent d'aborder l'histoire sociale, de présenter les Québécois comme des acteurs de leur histoire et de les personnifier. Une ligne du temps, disposée en hauteur tout au long du parcours chronologique, indique les événements majeurs internationaux, situant l'histoire du Québec dans le contexte élargi de l'histoire internationale. D'autres objectifs communicationnels s'expriment indirectement par le biais des thèmes sélectionnés, de l'approche choisie, du ton des textes et du choix des objets présentés.

Bien qu'ayant conscience qu'une exposition permanente s'adresse à tous les publics, l'équipe souhaitait privilégier le public québécois adulte en se basant principalement sur les références et les connaissances élémentaires de ce dernier. Elle devenait ainsi le complément de l'exposition permanente Nous, les Premières Nations, qui présente, comme son nom l'indique, l'histoire, 


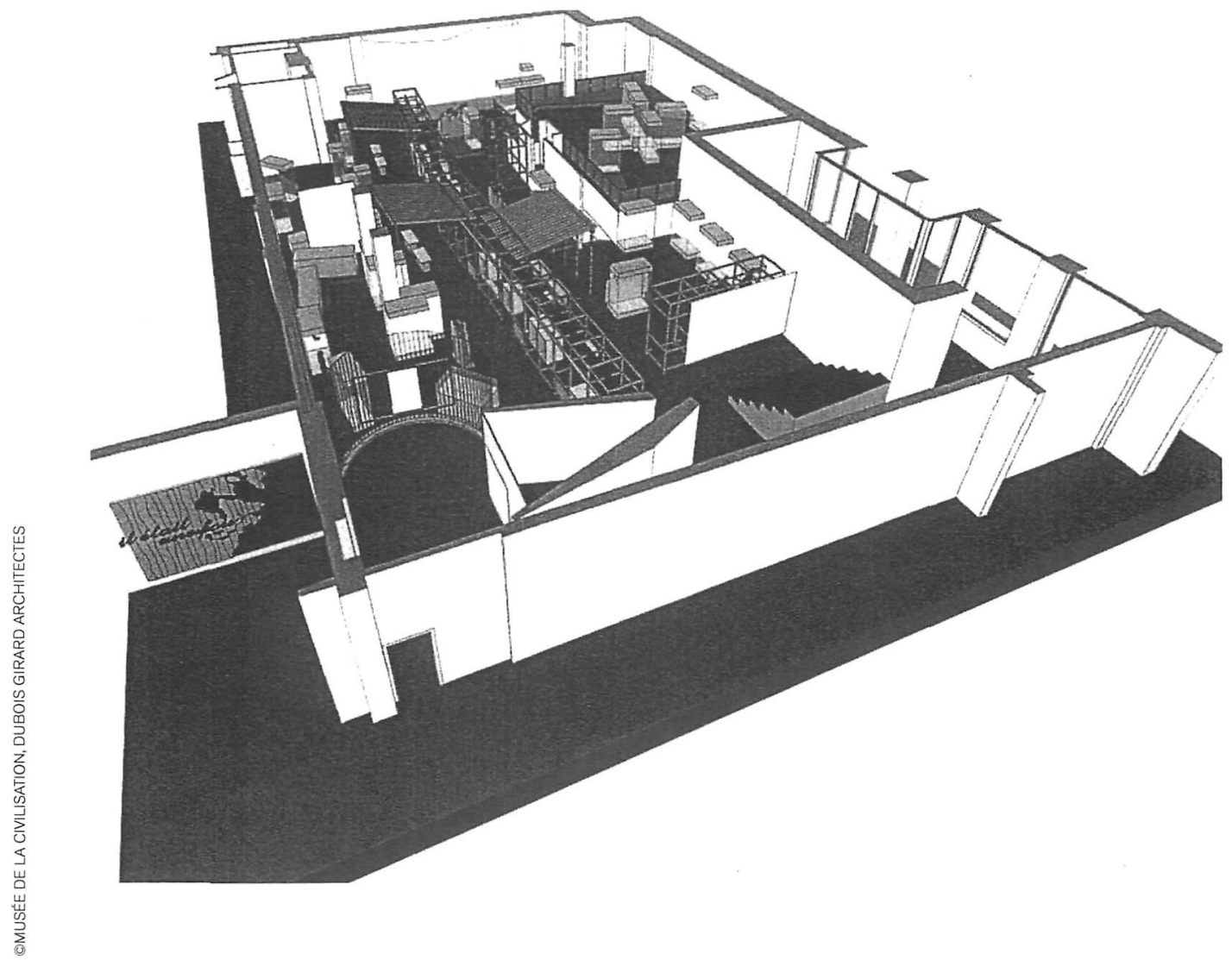

FIGURE 2

Vue en élévation du Temps des Québécois présentée dans le scénario daté du 29 octobre 2003, (Des modifications ont été effectuées comparativement à l'exposition actuelle, principalement dans l'entrée et à la fin du parcours, mais la structure globale est identique.) 
[8]

Ces films ne sont pas les films charnières évoqués ci-dessus, mais sont utilisés comme des objets.

[9]

Au service de la recherche et de l'évaluation du Musée de la civilisation.

[10]

Un questionnaire regroupant questions fermées et ouvertes guidait l'entrevue.

Cette entrevue durait en moyenne dix-sept minutes.

[11]

Idéalement, l'échantillonnage aurait dû être plus important mais cette recherche effectuée

dans le contexte du travail dirigé de la maîtrise en muséologie et les limites physiques et temporelles qu'il impliquait ne nous ont pas permis d'obtenir

un nombre de répondants supérieurs.

[12]

En raison de l'aspect synthétique de l'article, nous ne présentons pas ici tous les détails méthodologiques. Cependant, l'auteure reste disponible pour apporter au lecteur qui le souhaite des précisions complémentaires.

[13]

SPSS BASE 10.0, Chicago, États-Unis, C1999.

[14]

Les commentaires et les réponses ont été retranscrits sur traitement de texte avec un minimum de corrections.

Les réponses des visiteurs anglophones ont été traduites. L'analyse des comptes rendus a été effectuée de la façon suivante : nous avons fait des regroupements thématiques; pour certains nous avons mesuré la fréquence, puis nous

en avons fait une synthèse. Ces résultats s'insèrent progressivement dans les résultats statistiques que nous présentons, permettant souvent de préciser, de compléter et d'interpréter les données chiffrées. la culture et les enjeux contemporains des nations autochtones du Québec et qui est visitée principalement par un public touristique, étranger.

L'exposition s'organise selon une structure chronologique étoffée d'aspects thématiques. Quatre périodes se succèdent : « La Nouvelle-France, entre explorations et enracinement (1608-1754)", "Négocier la cohabitation (1763-1837)", "Révolutions et replis (1840-1960)", "C'est le début d'un temps nouveau (1960-2000)". Une charnière articule la transition entre chaque période et présente, par le biais d'un film, un événement clé ainsi que l'interprétation d'historiens. De chaque côté de cet axe central, des zones thématiques abordent la vie quotidienne, la religion par exemple. Pour chaque tronçon chronologique, les dispositifs rythment le parcours : panneaux, objets, cartels, films ${ }^{[8]}$, tiroirs vitrines, voix du passé...

\section{La méthode de travail}

Une fois le point de départ de l'exposition connu et analysé, il fallait comprendre la recomposition du visiteur. Cette recherche a été encadrée par André Allaire, responsable des études de public au Musée de la civilisation $^{[9]}$ : un questionnaire a été élaboré pour l'enquête, menée pendant treize jours aux mois de mai et juin 2005 auprès de 159 visiteurs adultes (plus de 18 ans). Les répondants, qui devaient avoir passé au minimum un quart d'heure dans la salle, étaient interrogés en entrevue face à face ${ }^{[10]}$, à leur sortie de l'exposition. Le taux de réponse s'établit à $58 \%$, ce qui assure une certaine validité aux résultats de l'enquête. Pour la taille de l'échantillon $(n=159)^{[11]}$, les réponses obtenues donnent des résultats et des indications significatifs ${ }^{[12]}$.

Les questionnaires complétés ont été codifiés et les réponses ont été saisies au moyen du progiciel SPSS base 10.0 pour Macintosh ${ }^{[13]}$, afin de produire des distributions de fréquences pour l'ensemble des questions fermées. Ces premiers résultats statistiques ont été complétés par l'analyse des réponses aux questions ouvertes et des commentaires des visiteurs sur certaines questions fermées ${ }^{[14]}$. Le croisement de certaines variables a été effectué pour affiner certains résultats. Le choix des tableaux croisés s'appuie sur la présence d'une relation statistique significative selon le test du chi carré (x 2, p $\leq 0,10)$. 


\section{Qui vient visiter cette exposition?}

Le portrait sociodémographique de notre échantillon présente un public majoritairement : féminin ( $56 \%$ ), âgé de 25 à 54 ans (71\%), actif (66\%), occupant un poste de cadre, de professionnel (53\%) ou de col blanc ( $21 \%)$, ayant fréquenté l'université $(68 \%)^{[15]}$, francophone $(64 \%)$. Il est à remarquer que la proportion entre Québécois ( $52 \%$ ) et non-Québécois (48\%) est équilibrée. Ce portrait est assez proche du public habituel du musée présenté lors des études de public annuelles estivales réalisées par André Allaire. Les écarts notables - l'origine géographique et l'âge des répondants - s'expliquent vraisemblablement par la période printanière où s'est effectuée l'enquête.

En termes d'antécédents à la visite, l'échantillon est majoritairement constitué d'habitués ${ }^{[18]}$ de ce type de loisirs $(79 \%)$. Toutefois, plus de la moitié $(58 \%)$ découvrent le Musée de la civilisation pour la première fois; ces nouveaux visiteurs se révèlent être non-Québécois (74\%). Principalement accompagnés ( $84 \%$ ), la majorité des visiteurs $(53 \%)$ comptent visiter plus de quatre expositions, lors d'un séjour que la plupart (61\%) planifient relativement court : de une à trois heures. La plupart des répondants $(79 \%)$ se disent intéressés par l'histoire, mais peu (11\%) sont venus spécifiquement pour visiter Le Temps des Québécois.

\section{[15]}

Fait à signaler, notre échantillon ne compte aucun répondant avec moins de huit années de scolarité.

[16]

Les visiteurs habitués des musées regroupent les visiteurs occasionnels (44\%) qui ont visité de un à trois musées dans les douze derniers mois et les amateurs ( $35 \%$ ) qui ont effectué plus de quatre visites dans ce même laps de temps. [ $\pi]$

ALLAIRE, André.

La provenance géographique mensuelle de la clientèle payante adulte du Musée de la civilisation, année 2005. Québec : Musée de la civilisation, Service de la recherche et de l'évaluation, mars 2006, p. 9.

Est-ce que le public réel est celui qui avait été privilégié par

l'équipe de conception?

TABLEAU 1

Provenance des visiteurs individuels du Musée de la civilisation en pourcentage, pour l'année $2005^{[17]}$

\begin{tabular}{|c|c|c|c|c|}
\hline 2005 & $1^{\text {ER }}$ TRIMESTRE & $2^{\mathrm{E}}$ TRIMESTRE & $3^{E}$ TRIMESTRE & 4E TRIMESTRE \\
\hline Québécois & $66 \%$ & $60 \%$ & $58 \%$ & $59 \%$ \\
\hline Non-Québécois & $34 \%$ & $40 \%$ & $42 \%$ & $41 \%$ \\
\hline
\end{tabular}


Pour une saison non touristique, et en comparant avec les données générales du Musée, la proportion des visiteurs étrangers dans l'exposition se révèle légèrement supérieure. Ce n'est donc pas le public québécois privilégié par l'équipe de conception qui semble le plus attiré par cette exposition. La caractéristique permanente de l'exposition peut expliquer en partie ce "désintérêt» : les Québécois ont le temps de visiter cette exposition. Les sujets abordés dans Mémoires, Nous, les Premières Nations et Le Temps des Québécois intéressent naturellement les visiteurs étrangers ou extérieurs à la province, puisqu'ils sont porteurs de l'identité du musée et de celle de la province, " pièces maîtresses du [...] mandat de diffusion et de conservation de la culture et de l'histoire du Québec et la signature du musée, sa vitrine $»^{[18]}$.

\section{Quelles sont les attentes des visiteurs de cette exposition?}

"Contempler des objets représentatifs de l'histoire du Québec » $(76 \%)$, [ 18] "acquérir de nouvelles connaissances " (69\%), "être sensibilisé(e) à l'histoire

Musée de la civilisation. Demande de subvention pour un renouvellement de deux expositions permanentes au Musée de la civilisation. Québec : Musée de la civilisation, 20 décembre 2001, p. 2. du Québec " $(63 \%)$ sont les principales attentes exprimées par les répondants. Les deux premières correspondent au comportement classique et attendu des visiteurs dans un musée. Les formulations « comprendre la spécificité de l'histoire du Québec » et « se divertir, avoir du plaisir " reçoivent un nombre moindre de suffrages ( $54 \%$ et $35 \%$ respectivement).

Les attentes valorisant l'acquisition de connaissances sur l'histoire québécoise et sa spécificité sont minorées par les répondants québécois, puisqu'ils affirment être déjà sensibilisés à cette histoire, notamment par le biais de l'enseignement scolaire, et profiter de cette visite pour se rafraîchir la mémoire.

En revanche, " contempler des objets emblématiques de l'histoire du Québec » est une attente exprimée par une majorité de Québécois (58\%). Les objets appartenant au passé, témoins d'une histoire nationale, symbolisent l'identité québécoise, l'ancrage matériel et affectif de cette histoire, évoquent des souvenirs, une mémoire commune que les visiteurs québécois viennent chercher. 


\section{Comment les visiteurs utilisent-ils et perçoivent-ils}

\section{l'espace d'exposition?}

Le temps consacré à la visite de cette exposition est relativement court au regard de sa superficie (670 mètres carrés). Près de 4 visiteurs ( $39 \%$ ) sur 10 prennent de 15 à 29 minutes pour parcourir l'exposition et un peu plus de 4 visiteurs $(42 \%)$ sur 10 y resteront de 30 à 59 minutes. Rester plus longtemps dans la salle est corrélé au fait d'être accompagné. La planification que les visiteurs font de leur séjour au musée et la concurrence directe des nombreuses expositions offertes au sein de l'institution expliquent en partie la rapidité de la visite.

De manière générale, la perception globale de la salle est bonne. Design, lettrage, son, éclairage, parcours rencontrent un fort taux de satisfaction $(80 \%$ et plus) auprès des répondants. Cependant, l'éclairage de la salle, le parcours et la taille des caractères cumulent les plus forts taux d'insatisfaction $(20 \%$, $16 \%, 10 \%$ respectivement). Les critiques vis-à-vis de l'éclairage de la salle et de la taille des caractères, souvent liées à l'âge du répondant, vont de pair. Si les faisceaux lumineux mettent en valeur les objets, les textes des panneaux et des cartels sont souvent considérés comme moins lisibles; un éclairage plus fort et un lettrage plus grand seraient appréciés.

Le parcours est le premier niveau de structuration de l'exposition et de ses messages. Si, dans Mémoires, le parcours était dirigé, pour Le Temps des Québécois un cheminement semi-dirigé a été choisi. Or, $16 \%$ des visiteurs s'en plaignent, le qualifiant d'étroit, de confus, de désorientant et de tortueux. Si la "colonne vertébrale» chronologique, politique et événementielle est identifiée, la relation avec les espaces périphériques abordant l'histoire sociale l'est moins : l'entrée, le sens de la visite, le « tournant " de la révolution industrielle semblent flous et des visiteurs entreprennent la visite à rebours. Les hésitations sur l'orientation diminuent d'autant l'attention portée au contenu. Le parcours apparaît ainsi le second élément gênant de l'espace d'exposition. Une grande partie des visiteurs apprécieraient un parcours dirigé ou préconisent le fléchage au sol.

Ily a donc lieu de se demander si le manque de lisibilité et de visibilité ainsi que la complexité du parcours influent sur l'utilisation de certains dispositifs et la réception des messages développés dans Le Temps des Quebécois. 
Comment les visiteurs utilisent-ils les différents dispositifs mis

à leur disposition et les apprécient-ils?

TABLEAU 2

Récapitulatif de l'utilisation et de l'intérêt des répondants aux dispositifs présents dans l'exposition

\begin{tabular}{|c|c|c|}
\hline DISPOSITIFS & $\begin{array}{l}\text { \% DES VISITEURS QUI ONT } \\
\text { UTILISÉ AU MOINS UNE FOIS... }\end{array}$ & $\begin{array}{l}\% \text { DES VISITEURS QUI SE DISENT } \\
\text { AU MINIMUM INTÉRESSÉS }\end{array}$ \\
\hline Objets & $100 \%$ & $98 \%$ \\
\hline Textes & $99 \%$ & $93 \%$ \\
\hline Films & $71 \%$ & $92 \%$ \\
\hline Tiroirs vitrines & $38 \%$ & $84 \%$ \\
\hline Vitrines enfants & $45 \%$ & $57 \%$ \\
\hline « Voix du passé " & $60 \%$ & $71 \%$ \\
\hline
\end{tabular}

L'ensemble des dispositifs présents dans la salle sont identifiés et utilisés. Plus précisément, " observer les objets», «lire les textes», " regarder les films " sont les activités les plus fréquentes dans le comportement des

[19]

MACDONALD, Sharon *Un nouveau * corps de visiteurs"; musée et changements culturels». Publics et musées, Du public aux visiteurs, $n^{\circ} 3$, juin 1993, p. 21.

[20]

Les visiteurs accompagnés d'enfant(s) et les familles sont sous-représentés dans notre échantillon, du fait de la durée de l'entretien (15-20 minutes).

Le volume des données recueillies est trop faible pour effectuer une analyse fiable.

Seulement neuf visiteurs interrogés étaient accompagnés d'enfant(s) de moins de douze ans. Cinq d'entre eux ont remarqué et utilisé les vitrines. visiteurs interrogés, tandis que "écouter les voix du passé», " ouvrir les tiroirs vitrines ", « regarder les vitrines destinées aux enfants " sont les moins utilisées.

En somme, les principaux dispositifs de l'exposition sont logiquement les plus utilisés par les visiteurs, comportement sans doute renforcé également par la force de l'habitude ${ }^{[19]}$ : de nombreux visiteurs disent privilégier les textes et les objets, vecteurs efficaces et clés du propos, lorsque leur temps de visite est limité. Finalement, Les visiteurs non québécois, probablement curieux et motivés par leur désir de connaître l'histoire du Québec, estiment lire un plus granḍ nombre de textes que les Québécois.

Quant aux vitrines destinées aux enfants, leur discrétion et la sousreprésentation du public jeune et familial auquel elles s'adressent justifient leur faible utilisation ${ }^{[201}$. Quand elles sont remarquées, elles sont identifiées comme une activité marginale au sein de l'exposition.

Les bornes d'écoute des "voix du passé" s'avèrent contraignantes dans leur mode de fonctionnement. Chaque pupitre offre deux casques d'écoute 

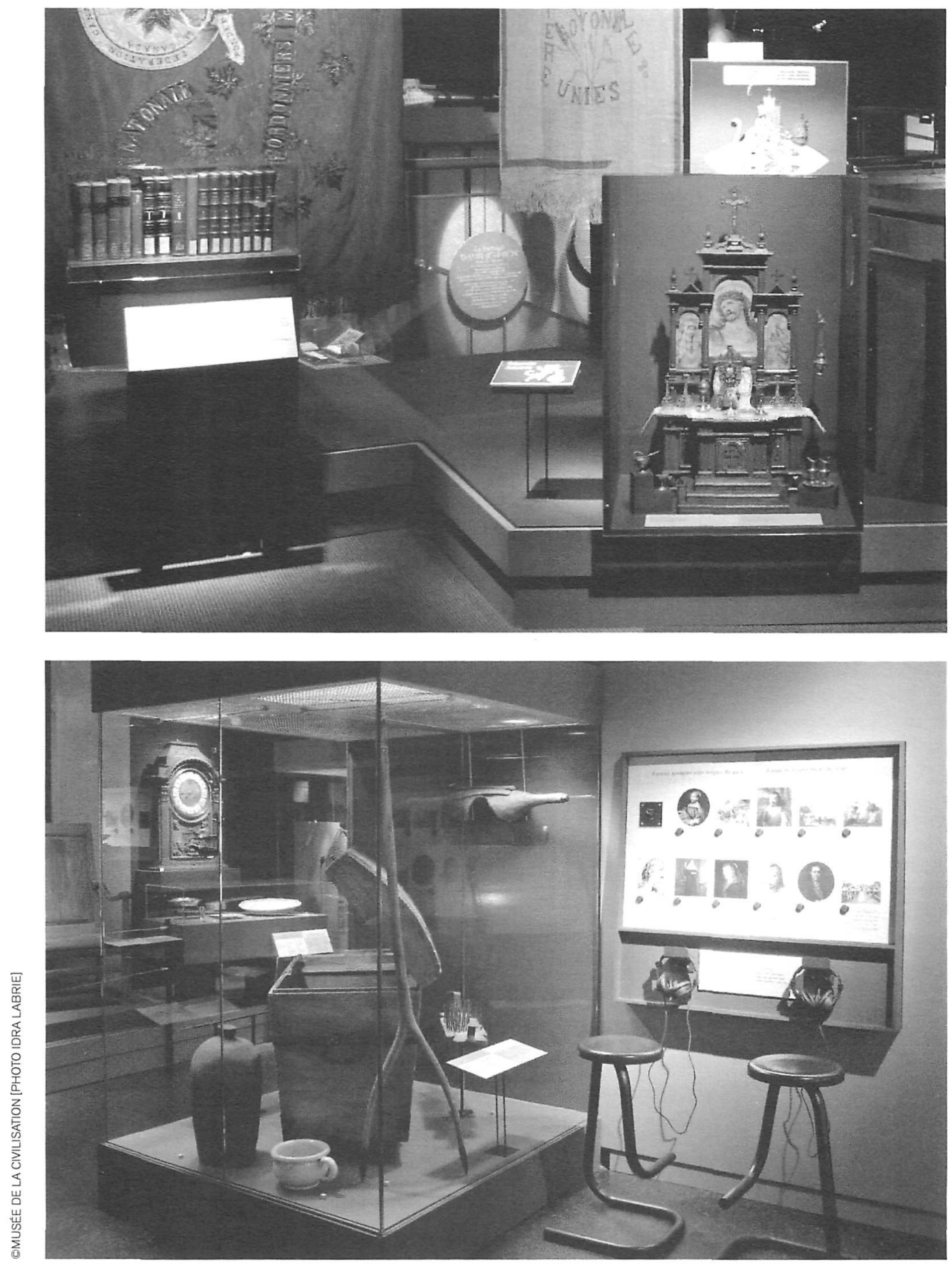

FIGURE 3

Exemple d'une vitrine destinée aux enfants dans Le Temps des Québécois.
FIGURE 4

Exemple du dispositif "les voix du passé". 
dépendants et chaque témoignage enclenché doit être écouté entièrement avant d'en changer. Les visites scolaires étant nombreuses, ces dispositifs sont souvent pris d'assaut par les élèves, laissant peu l'occasion aux visiteurs adultes libres de les utiliser.

L'analyse de l'intérêt des visiteurs peut également expliquer les fréquences d'utilisation. Les objets, les textes, les tiroirs vitrines et les films cumulent le plus fort taux d'intérêt (80\% et plus) ${ }^{[21]}$.

[21]

Les intérêts et l'appréciation des visiteurs s'appuient sur les réponses ouvertes données par les répondants.

[22]

II y a $39 \%$ des visiteurs qui citent les objets comme élément préférés dans l'exposition.

[23]

BERGERON, Yves. "De la notion privée d'héritage matériel au concept universel et extensif de patrimoine", Actes du colloque médias et patrimoine. Le rôle et l'influence des médias dans la construction d'une mémoire collective. Québec : Institut sur le patrimoine culturel, Université Laval, 2003, p. 45-52. Texte en réponse à la communication d'André Desvallées, «De la notion privée d'héritage matériel au concept universel et extensif de patrimoine : retour sur l'histoire et sur quelques ambiguités sémantiques", p. 19-35. Cette publication est disponible en format PDF sur le site <www.ulaval.ca/ipac/>.

[24]

Le mousquet des premiers trappeurs a interpellé plus d'un visiteur!

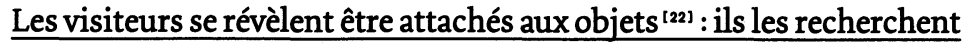
vieux, authentiques, emblématiques, rares; le musée n'est-il pas par définition le gardien de trésors et d'objets exceptionnels ${ }^{[23]}$ ? Ayant appartenu à des personnes importantes pour l'histoire du Québec, ils deviennent témoignages de cette époque et de ces personnages. D’autres, illustrant la vie quotidienne, rendent concrets des modes de vie passés ${ }^{[24]}$. De nombreux Québécois découvrent des objets inconnus, uniques, surprenants, qualificatifs accentués lorsqu'ils sont enchâssés dans des tiroirs vitrines. Efficace, ce dispositif donne aux visiteurs le sentiment qu'ils sont privilégiés; il met en exergue leur fragilité, leur délicatesse. Enfin, les objets illustrant le passé récent émeuvent particulièrement les visiteurs québécois : la première carte de sécurité sociale, le casse-tête plouf... Ces artéfacts connus, possédés, manipulés, évoquent alors des souvenirs personnels et donnent la sensation d'appartenir à l'histoire du Québec ou d'y avoir participé.

Les textes sont appréciés pour leur concision et leur didactique. Bien situés et élaborés, étayés de dates et de cartes, ils introduisent efficacement les différentes parties de l'exposition. Les cartels sont clairs, détaillés et explicatifs. Parfois quelques objets mériteraient un cartel avec davantage d'explications.

Les vitrines destinées aux enfants et les "voix du passé" cumulent le plus fort taux d'indifférence et de déception ( $29 \%$ et $43 \%$ respectivement). Comme indiqué précédemment, la sous-représentation du public ciblé explique cette perception négative. Les adultes interrogés disent avoir remarqué ces vitrines, sans comprendre la consigne. Quelques-uns trouvent que le jeu ne suscite pas suffisamment la curiosité. Cette mauvaise appréciation peut limiter le rôle de prescripteur des visiteurs adultes libres auprès du public familial ciblé.

Les "voix du passé» sont appréciées par une majorité (71\%) d'utilisateurs, puisqu'elles rendent l'histoire plus humaine, vivante, proche. Les critiques formulées relèvent cependant le manque de fonctionnalité du dispositif : nombre limité de casques d'écoute, longueur et surabondance de témoignages... Le manque d'authenticité déçoit les visiteurs : des voix réelles pour les témoignages, lorsque possible, seraient préférables aux voix d'acteurs. L'authenticité porteuse d'émotions que la reproduction ne peut 
égaler est un critère important et attendu de la part du public, quelles que soient les formes «d'objets » exposés au musée.

\section{Précisions sur les dispositifs vidéo :}

\section{utilisation, perception générale et spécifique à l'exposition}

Le médium vidéo est important dans cette exposition : soit le film est utilisé comme un objet, témoignage d'une époque, d'un événement, illustrant un propos; soit construit pour l'exposition, il articule le discours et, à l'image d'un panneau, il supporte une partie conséquente du message développé. Cette deuxième utilisation des films a été analysée plus finement, du fait qu'ils rythment le parcours en tant qu'introduction, conclusion et transition. Bien que ce soit le troisième dispositif le plus utilisé par les visiteurs, des distinctions d'utilisation doivent être faites, selon la situation du film, sa longueur et le visiteur.

La majorité des visiteurs (60\% et $62 \%$ ) ne regardent ou ne remarquent pas les films d'introduction et de conclusion. L'emplacement du film d'introduction semble être problématique : immédiatement à gauche en entrant, avant le grand écran - signal d'appel, les visiteurs passent souvent à côté, attirés par les premiers objets et les panneaux, seconds ${ }^{[251}$ repères lumineux d'une entrée plutôt sombre. De plus, ce petit écran est équipé de deux combinés d'écoute et les visiteurs qui le regardent forment un barrage et le masquent aux visiteurs qui pénètrent dans l'exposition, portés alors à commencer la visite par la fin.

Le film conclusif est remarqué ( $93 \%)$, mais moins regardé $(53 \%)^{[26]}$. Le grand écran est enclos dans un espace muni de gradins, mais la fatigue, la lassitude vis-à-vis de ce média, la longueur ${ }^{[27]} \mathrm{du}$ film, l'arrivée à la fin de la projection et l'inconfort des bancs sont autant d'explications invoquées par les personnes interrogées pour expliquer leur manque d'intérêt.

De façon générale, la plupart des visiteurs (79\%) apprécient ce type de médium dans les expositions ; l'image fait suffisamment partie de notre société pour être admise au musée par une large part des répondants. Ils sont alors qualifiés de modernes, efficaces, didactiques, conviviaux, concrets; ils complètent le discours, rendent vivant l'espace d'exposition, constituent une alternative à la lecture, rompent la monotonie, permettent de se reposer. Leurs opposants, redonnant au musée son rôle de gardien d'objets extraordinaire, trouvent qu'ils n'ont pas leur place dans un musée, puisque c'est un objet de leur quotidien. Souvent trop longs, les films les rendent

\section{[25]}

Le premier repère lumineux est le grand écran servant de signal d'appel.

[26]

À titre comparatif, le film d'introduction est remarqué par $83 \%$ des visiteurs interrogés et $45 \%$ des répondants ne l'ont pas regardé du tout. [27]

La durée totale du film est affichée sur un panonceau à l'entrée de la «salle de visionnement». 
inactifs et divulguent trop rapidement l'information pour qu'ils puissent s'en souvenir.

Les films articulant le propos de l'exposition intéressent la plupart des visiteurs (92\%). Bien faits, ils remettent les événements dans leur contexte, décrivent leurs conséquences, montrent d'autres opinions, soulèvent des interrogations, expliquent bien l'évolution des Québécois, résument le

Le Québec et ses historiens, de 1840 à 1920. Québec : Université de Laval, 1978 p. $415-423$ squelette de l'exposition, rendent l'histoire plus vivante et permettent de la comprendre aujourd'hui. L'intervention d'historiens, signe d'authenticité et de scientificité, est particulièrement appréciée. Ce sentiment serait-il, de la part des Québécois, une trace de l'autorité que possédaient les historiens cléricaux, détenteurs d'un pouvoir social et intellectuel conséquent il y a encore peu de temps ${ }^{[281}$ ? Les critiques recueillies portent surtout sur la fonctionnalité : longueur, grand nombre... Le peu de temps prévu pour visiter l'exposition oblige le visiteur à faire des choix et les films semblent être les premiers à en faire les frais.

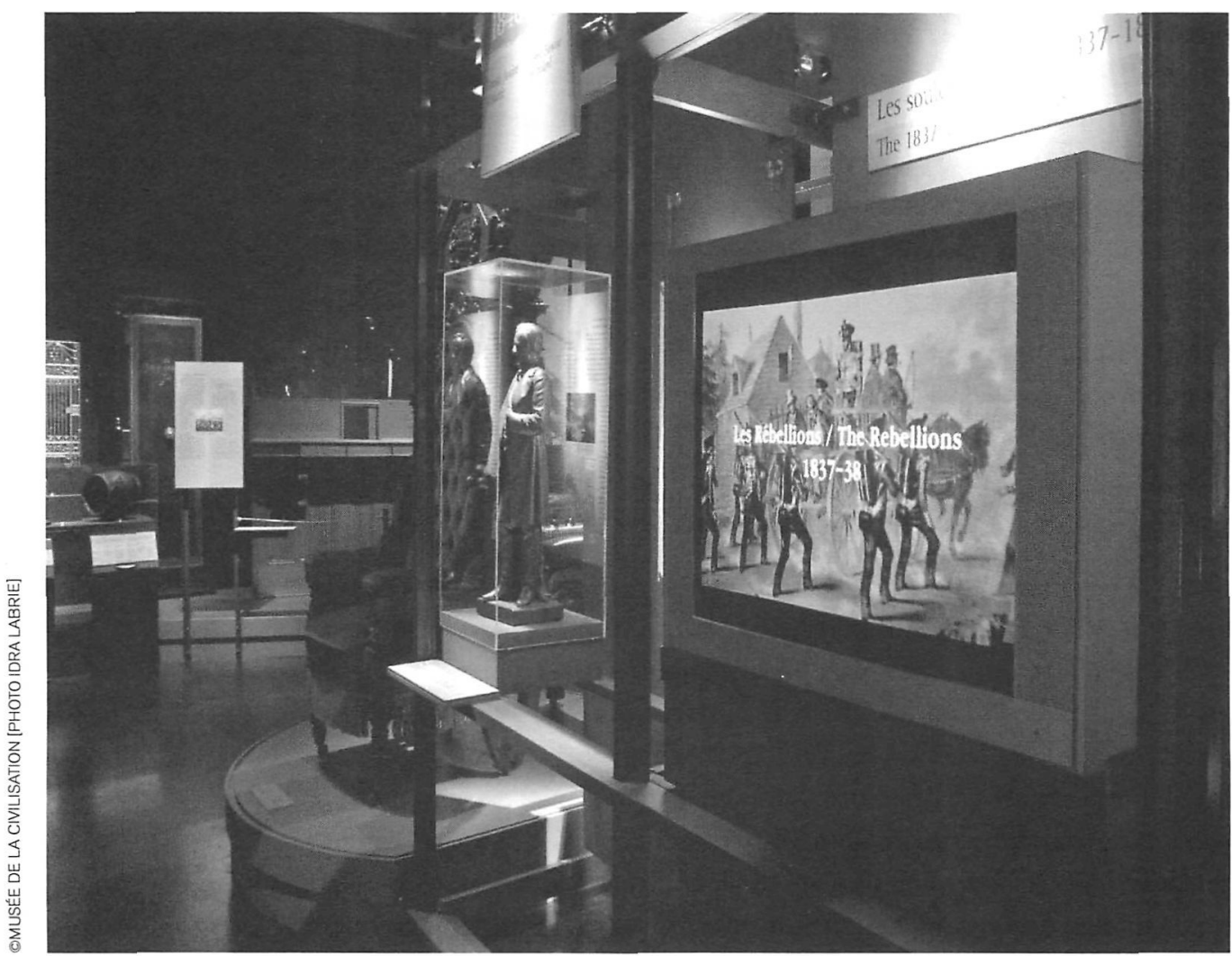


Finalement, l'authenticité, l'émotion suscitée, l'aspect didactique et l'efficacité marquent les dispositifs préférés des visiteurs, tandis que les contraintes limitent leur utilisation. La diversité des dispositifs est appréciée, mais allonge d'autant la visite, s'opposant ainsi à l'idée que le visiteur se fait de son séjour au musée. Pour certains répondants, l'exposition nécessite du temps, demande que l'on y revienne ou doit être vue dès le début de la visite au musée. La complexité du parcours semble n'avoir d'impact que sur l'utilisation et l'identification du film d'introduction.

L'exposition parcourue, les différents dispositifs plus ou moins utilisés permettent-ils au visiteur de recomposer les intentions communicationnelles de l'équipe de conception?

Les visiteurs recomposent-ils les messages de l'exposition?

De façon générale, les messages principaux de l'équipe de conception ont bien été identifiés par les visiteurs.

TABLEAU 3

Pourcentage d'adhésion que les différents énoncés ont recueilli

\begin{tabular}{llc}
\hline ÉNONCÉS PROPOSÉS & $\begin{array}{l}\text { TOUT À FAIT D'ACCORD ET } \\
\text { PLUTÓT D'ACCORD }\end{array}$ & $\begin{array}{l}\text { PAS D'ACCORD ET PAS } \\
\text { D'ACCORD DU TOUT }\end{array}$ \\
\hline $\begin{array}{l}\text { Cette exposition met en valeur des } \\
\text { épisodes du passé dont les enjeux } \\
\text { sont encore importants aujourd'hui }\end{array}$ & $94 \%$ & $4 \%$ \\
$\begin{array}{l}\text { Cette exposition présente une } \\
\text { synthèse de l'histoire des Québécois }\end{array}$ & $95 \%$ & $5 \%$ \\
$\begin{array}{l}\text { Cette exposition montre la vie } \\
\text { des Québécois }\end{array}$ & $90 \%$ & $10 \%$ \\
$\begin{array}{l}\text { Cette exposition fait réfléchir } \\
\text { sur l'histoire du Québec }\end{array}$ & $87 \%$ & $13 \%$ \\
\hline $\begin{array}{l}\text { Cette exposition donne une vision } \\
\text { positive de l'histoire du Québec }\end{array}$
\end{tabular}


[29]

Les périodes oubliées citées lors de ces commentaires sont la période industrielle, la Seconde Guerre mondiale et après 1970 .

[30]

GAGNON, op. cit., p. 418

[31]

Notamment la relation entre l'espace chronologique central plutôt politique et les espaces sociaux périphériques.

[32]

En effet, $68 \%$ des commentaires recueillis affirment que l'exposition propose une vision de l'histoire objective et $18 \%$ trouvent que l'histoire est de toute façon négative.
L'utilisation de certains dispositifs semble être corrélée avec la compréhension de plusieurs messages et elle les améliore parfois. L'exposition fait réfléchir sur l'histoire de la province lorsque les visiteurs ont utilisé l'ensemble des dispositifs de l'exposition, ce qui sous-entend une visite relativement longue et approfondie. «Regarder les films charnières " favorise la mise en valeur des épisodes passés actuellement prégnants; « regarder le film conclusif " aide à percevoir l'aspect synthétique de l'exposition et "écouter les 'voix du passé’ » à montrer la vie des Québécois.

Les Québécois expriment plus fréquemment leur désaccord que les visiteurs " étrangers". Ayant un minimum de connaissances de leur histoire, lis en ont une perception, peut-être nationaliste ou fédéraliste, qui ne correspond pas forcément à celle présentée dans l'exposition, alors que les visiteurs non-Québécois, néophytes, n’ont ni les connaissances, ni le parti pris, ni la perception globale pour interroger la vision qui leur est proposée.

Les Québécois réagissent surtout (malgré le faible taux de désaccord) sur l'aspect synthétique. Plusieurs d'entre eux estiment que l'histoire ne peut être synthétisée dans un espace alors considéré comme petit, restreint, ou par un nombre d'objets limité. La synthèse est alors jugée partielle, rapide, superficielle, éparpillée, des événements sont oubliés ${ }^{[20}$. D’autres, conscients de ne pas avoir tout vu, modèrent leur adhésion, en raison d'une perception partielle, tronquée de l'exposition.

À l'inverse du parti pris de Mémoires, l'histoire politique et événementielle supplante la vie des Québécois. En ce sens, cette présentation constitue un retour vers une historiographie plus classique ${ }^{[30]}$. Les visiteurs déclarent que la vie des Québécois est traitée de façon superficielle, générale ou, si elle est détaillée, elle se révèle alors fragmentaire, limitée à une époque ou à une partie de la population (la révolution industrielle, les personnes importantes qui ont influencé la vie des Québécois, les immigrants, les «indigènes»). Reprenant des thématiques présentées dans Mémoires, quelques visiteurs expliquent qu'il faudrait aborder le folklore, les fêtes religieuses, mettre en valeur le mode de vie, la vie rurale, l'importance des saisons et notamment de l'hiver pour mettre en relief la vie québécoise. Le fait de trouver le parcours complexe ${ }^{(311}$ ou ne pas avoir écouté les "voix du passé» influence également la compréhension du message.

L'aspect réflexif de l'exposition est assez atténué par les répondants québécois : elle suscite des émotions, rafraîchit la mémoire. Plutôt factuelle, elle donne envie d'approfondir des thèmes. Enfin, la vision de l'histoire proposée est jugée objective, neutre, réaliste, nuancée, impartiale; le ton est explicatif et informatif. Peu de visiteurs la jugent négative ou triste ${ }^{[32]}$. L'analyse des commentaires montre que l'exposition ne bouscule pas la 
vision ni les connaissances des Québécois : le point de vue de l'exposition, suffisamment consensuel, correspond au leur.

Globalement, l'exposition atteint ses objectifs en termes d'utilisation des dispositifs et de recomposition des contenus, bien que la complexité du parcours et la faible utilisation des " voix du passé » semblent amoindrir l'aspect social de l'exposition.

L'expérience de visite est satisfaisante,

mais l'exposition sera peu revisitée

TABleaU 4

Degré d'appréciation de l'exposition des répondants en pourcentage.

\begin{tabular}{ll}
\hline$\%$ DES VISITEURS INTERROGÉS & DEGRÉ D'APPRÉCIATION \\
\hline 47 & l'exposition a beaucoup plu \\
\hline 47 & l'exposition a plu \\
\hline 1 & indifférent \\
\hline
\end{tabular}

LeTemps des Québécois plaît à la plupart des visiteurs (94\%). Selon l'analyse des coefficients de corrélation simple, plusieurs facteurs sont reliés positivement à la satisfaction :

- $\quad$ être non-Québécois ${ }^{[33]}$

- $\quad$ être Montréalais

- $\quad$ effectuer sa première visite au musée

- ne pas visiter seul(e)

- visiter longuement l'exposition

- $\quad$ lire les textes

- regarder les films « contenu» de l'exposition

[33]

Cette variable est confortée par l'enquête de fréquentation et d'appréciation des expositions de l'été 2005 du Musée de la civilisation, menée par André Allaire : «le fait d'être Québécois diminuerait en soi les chances de choisir Le Temps des Québécois comme étant l'exposition la plus intéressante au musée.

À l'inverse, être Français accroît significativement les chances de mentionner cette exposition comme étant la préférée. Donc, nous pouvons affirmer que les Français représentent le public type de notre plus récente exposition permanente." (ALLAIRE, André. La fréquentation et l'appréciation des expositions de l'été 2005 du Musée de la civilisation, Rapport final. Québec : Musée de la civilisation, Service de la recherche et de l'évaluation, novembre 2005, p. 13.)

- regarder en entier le film de la conclusion

- comprendre les messages de l'exposition 

confirmée lors de l'enquête estivale 2006 : «L'un des déterminants sociodémographiques du fait d'avoir choisi

Le Temps des Québécois comme exposition la plus intéressante est de ne pas résider au Québec." (Idem, p. 10-14.)

[35]

Si nous avons posé la question à tous les visiteurs, nous ne prenons volontairement en compte ici que les Québécois. Certains visiteurs canadiens ou étrangers ont répondu affirmativement, mais cela exigerait qu'ils reviennent au Québec... À titre indicatif, si nous prenons la totalité de l'échantillon, la proportion est quasiment identique (47\%).
Les résultats précédents basés sur les corrélations confirment les pistes identifiées au cours de l'analyse des résultats. L'exposition satisfait naturellement l'intérêt des non-Quebécois ${ }^{[34]}$ et des nouveaux visiteurs. Elle semble également combler les désirs des visiteurs accompagnés. La diversité des médias facilite sans doute la visite à plusieurs, en multipliant les occasions d'interaction.

Comprendre les messages véhiculés par l'équipe de conception est un élément important de satisfaction. Pour cela, rester longuement, prendre le temps de regarder les films, notamment celui de la conclusion, et lire plusieurs textes facilitent la compréhension et favorisent donc la satisfaction.

La variable montréalaise est difficilement explicable ici. Une étude plus fine sur la réception et la perception des Québécois serait probablements nécessaire. Une telle étude révélerait sans doute pourquoi l'exposition satisfait moins les habitants de la capitale nationale que ceux de la métropole : est-ce parce que les objets ont déjà été vus par les Québécois ? Est-ce parce que la ville de Québec avec son centre historique regorge de plaques commémoratives, de sites, de musées et de références à la Nouvelle-France? Ses habitants immergés dans cette présence historique seraient-ils moins curieux, moins intéressés par une exposition sur ce thème? Ce creux, ce vide, mettrait-il alors en exergue la curiosité, l'intérêt des Montréalais issus d'horizons géographiques différents, moins familiers de cette histoire?

Une très grande majorité des répondants $(96 \%)$ recommanderaient l'exposition. En revanche, il est intéressant de noter qu'en termes de "revisite", seulement $48 \%$ des visiteurs québécois ${ }^{\text {(35) }}$ pensent revenir la voir s'ils ont « de la visite " ou avec les enfants. Plusieurs la conseilleraient aux touristes, aux élèves en histoire. Son aspect instructif, didactique est clairement identifié. Le côté factuel du Temps des Québécois, la vision historique peu novatrice, le peu d'émotions qu'elle suscite contrairement à l'exposition Mémoires expliquent probablement cette faible volonté de la revisiter. 
L'exposition Le Temps des Québécois, héritière de Mémoires?

Plus d'un tiers des visiteurs interrogés ( $36 \%$ ) ont visité les autres expositions permanentes du musée. Mémoires arrive en première position (38\%) dans l'ordre de préférence, suivie du Temps des Québécois (33\%) et de Nous, les Premières Nations (29\%):

Le Temps des Québécois est en bonne position. Si de nombreux visiteurs préferent encore Mémoires (1988-2005), cette dernière a été présentée suffisamment longtemps pour que les visiteurs québécois se l'approprient. L'aspect émotif de l'exposition favorise cette identification québécoise et satisfait également la curiosité des visiteurs non québécois ${ }^{[36]}$. Les résultats des enquêtes de fréquentation et d'appréciation des expositions des étés 2005 et 2006, menées par André Allaire, montrent que Le Temps des Québécois appartient au groupe d'expositions appréciées par les visiteurs. À l'été 2006, après la disparition de Mémoires, Le Temps des Quebécois devient l'exposition permanente préférée, mais connaît une diminution de l'intérêt exprimé par les visiteurs ${ }^{[371} \ldots$

Une exposition didactique, mais qui " fonctionne " différemment selon les publics

Globalement, Le Temps des Quebécois fonctionne bien : malgré un parcours complexe, les dispositifs sont identifiés et utilisés, les messages que l'équipe de conception voulait transmettre sont effectivement recomposés par les visiteurs. En dépit des éléments de confort qui doivent être améliorés, l'expérience de visite se révèle, dans son ensemble, satisfaisante.

Dans le contexte de séjour propre au Musée de la civilisation - multiples visites en un temps limité -, les dispositifs fortement contraignants comme les films ou les "voix du passé» restreignent le comportement du visiteur et par là même leur auditoire, à la différence des textes ou des objets. Pour atténuer ce phénomène, une réflexion devrait être menée afin d'alléger les modalités d'utilisation et d'améliorer la perception du contenu dont ils sont porteurs.

Finalement, le public québécois privilégié lors de la conception ne semble pas être le public naturel de la nouvelle exposition permanente. La motivation de la visite, la perception des messages et la satisfaction sont influencées par la provenance des visiteurs.
[36]

SAUVAGE, Cécile.

Mémoires au Musée de la civilisation de Québec.

Regards croisés sur une exposition permanente. Québec : Musée de la civilisation, Les cahiers du Musée de la civilisation, 2004, p. 69.

[37]

À l'été 2005, 20\% des visiteurs étaient intéressés par cette exposition; en 2006, cette proportion était de $15 \%$. Cette baisse peut-elle s'expliquer par la programmation différente entourant Le Temps des Québécois? (ALLAIRE, La fréquentation et l'appréciation des expositions de l'été 2006 ..., op. cit, p. 7 et 9.) 


\section{TABLEAU 5}

Résumé de la perception de l'exposition selon la provenance des publics

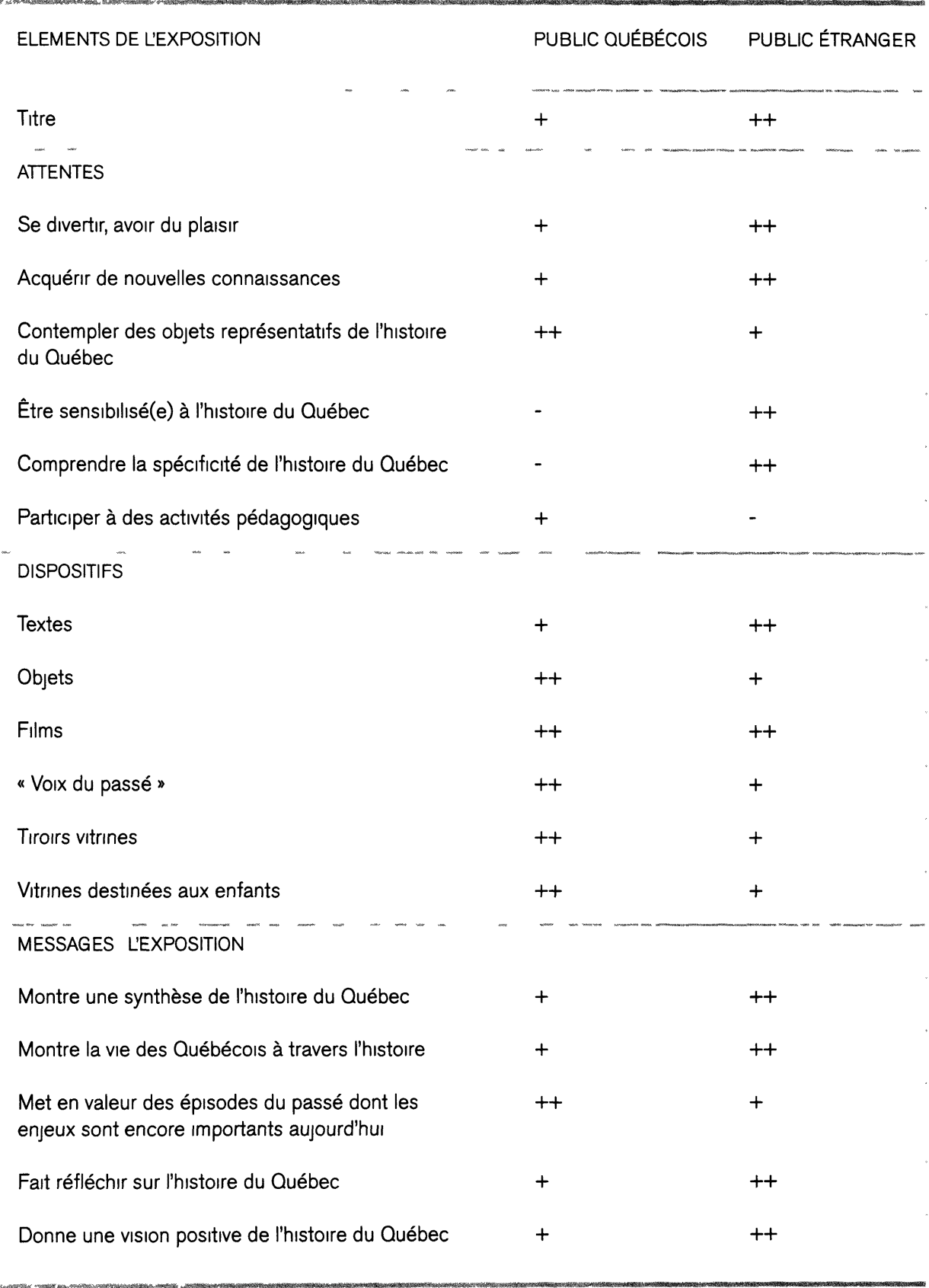

+ : ce qui attire ou suscite l'intérêt du public.

- : ce qui n'attire, n'intéresse pas ou peu le public. 
Effectuer une enquête de public en période hivernale permettrait sans doute d'affiner la perception et la réception des Québécois et du bassin de clientèle locale, de la capitale.

Si le bilan en termes de recommandation et de revisite s'équilibre, la variété et la densité des dispositifs, jumelées à un contexte de visite propre au Musée de la civilisation, donnent un goût d' " inachevé " à de nombreux visiteurs. Effectivement, ceux-ci viennent au musée dans l'optique de visiter un maximum d'expositions, limitant de fait la durée de visite de cette salle d'exposition. La richesse médiatique participera sans doute à sa " revisite ", même si l'on est en droit de s'interroger sur le rôle que peut jouer le public québécois comme maillon dans la chaîne de réception de l'exposition ${ }^{[38] . . .}$

Effectivement, le public québécois moindrement attiré par cette exposition exprime peu d'attentes. Si les objets sont des éléments importants dans leur expérience muséale, l'exposition ne modifie pas leur perception del'histoire du Québec, ne suscite pas de réflexion particulière à ce sujet, ce qui explique sans doute leur faible intention de la revisiter. Pour ces visiteurs, l'exposition a un intérêt essentiellement touristique et pédagogique. Si le facteur émotif était sans doute un élément important et explicatif du succès et de l'appropriation de Mémoires, l'avenir nous dira si l'aspect didactique sera celui du Temps des Québécois.

\section{[38]}

Lors de l'enquête de fréquentation et d'appréciation des expositions de l'été 2005 , menée par André Allaire, une baisse de la fréquentation a d'ailleurs été observée pour Le Temps des Québécois, passant de $72 \%$ en août 2004 à $53 \%$ en août 2005 , baisse qui peut être expliquée *par le fait que [...] le public était beaucoup plus régional cet été qu'en 2004 ». (Idem, p. 6.) 


\section{Summary}

\section{Le temps des Québecois}

From a communication motive to its reception.

[translated by Laurence Duval-Teasdale]

An exhibition is the spatial, tridimensional structuring of an attempt to communicate ideas and messages from a designer/communicator to the public. Accordingly, the designer builds an organized space and creates an itinerary which will communicate the message during the exhibition. This structure is done with the help of devices combining artifacts, media and interpretative supports, which will effectively communicate the content. ${ }^{[39]}$ For this purpose, the designer thinks of a standard visitor, however this changes when the exhibit starts. This is when the real visitor

This definition comes from the class MSL 6104 "Exposition, interpretation et diffusion", given by MM. Bernard Schiele and Raymond Montpetit. comes into the action and restores the spatial rebus that is the exhibit. He may use the devices, change them from their original position, examine a special part of the message, and change the meaning...

It is this problematic, this re-setting that we have tried to understand while doing the summative evaluation of Le Temps des Québécois. Disciplinarian and time-sequential, the new permanent exhibit at the Musée de la civilisation is the first of its kind at the Museum. It started about a year ago, but now the inauguration excitement is over. Who is now visiting this exhibit? How do they seize the itinerary? Do they use the different supports -in particular the movies- that are furnished? How do they take over and perceive the exhibit Le Temps des Québécois? Do they reset and appropriate the communicational intention exposed in the exhibit? Is the exhibit and the experience satisfying for the visitors? If not, why?

To answer these questions, we have done an analysis of the messages and the space of the exhibition. Then, we elaborated a questionnaire including opened and closed questions, in order to get more precise and explicative results. We conducted the survey within 13 days, with face-to-face interviews while the visitors were leaving the exhibition. 159 visitors replied. They had to be older than 18 , and have visited the exhibition for more than 15 minutes.

The socio-demographic portrait of our sample is somewhat the same as the usual museum attendance: composed from as many Quebecers as non-Quebecers, 
most of the respondents are a museum clientele and say they are interested by History. However, they are not at the museum especially for this exhibition, but for the whole institution. In this way, they intend to visit as many exhibitions as they can. This behavior shortens the time they spend to each exhibition, in particular this room.

Concerning the exhibition space, the overall perception is good. However, the comfort level for reading and object observation -lightning and size of the script type- can be improved. On the other hand, the visitors think the itinerary is complex, long, and thus the peripheral spaces are derelict. The circulation limits their appreciation of the visit. The devices are identified, used and their diversity is appreciated: the objects, the displays for their efficiency, the synthetic and didactic aspects. However, the children's displays are not perceived by most of the visitors and the operation is misunderstood. The audio testimonies are less efficient because of their restrictive, non-functional aspects. Despite these few space and device malfunctions, they don't seem to interfere with the resetting of the messages. Nevertheless, the Quebecers, judges and parties, are naturally more critical on the synthesis proposed by the exhibition. Perceived objectively and in a politically correct fashion, the exhibition generates few questions and little thinking, but refreshes the knowledge of the Quebecer public. It is identified as an efficient pedagogic tool and will be widely recommended by its visitors, even though if they won't go back... 